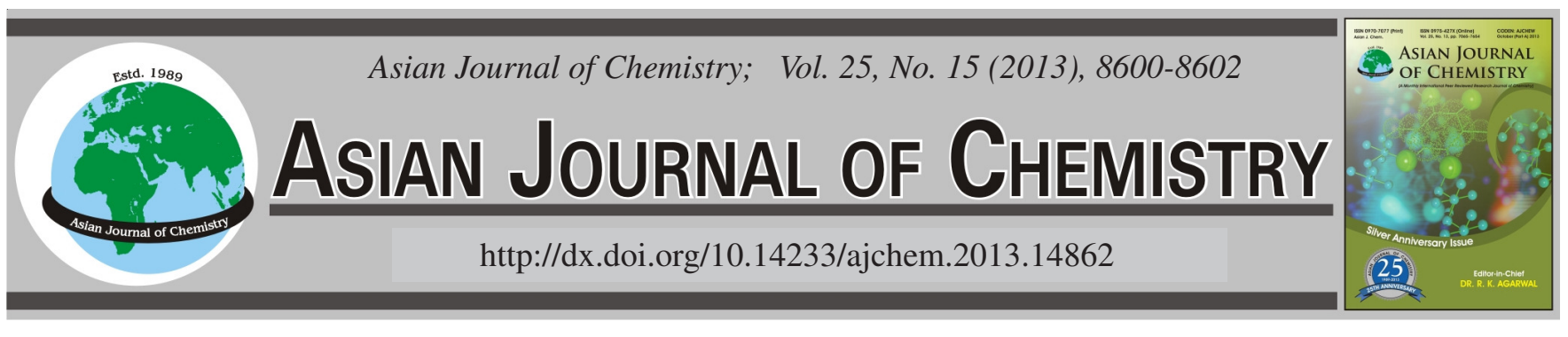

\title{
Structure-Activity Relationship of Diacylhydrazine Derivatives Fluoride-Containing Pyrazolyl Moiety
}

\author{
Hui-Min Bi ${ }^{1}{ }^{*}$, Peng-Tao XiE ${ }^{1}$, Shu-XIAn Li ${ }^{1}$, Yong-Li Li ${ }^{1}$ and Chun-Hua Dong ${ }^{1,2}$
}

${ }^{1}$ Handan Key Laboratory of Organic Small Molecule Materials, Handan College, Hebei Handan 056002, P.R. China

${ }^{2}$ Institute of Chemistry, Chinese Academy of Sciences, Beijing 100190, P.R. China

*Corresponding author: E-mail: binbi99@163.com

(Received: 23 November 2012;

Accepted: 26 August 2013)

AJC-14005

\begin{abstract}
Diacylhydrazine derivatives fluoride-containing pyrazolyl moiety compounds were studied by Gaussian03. They have good herbicidal activity. The optimized geometries were calculated and the information of their electron density of these compounds and energy levels, main composition and proportion of the frontier orbitals were obtained by the HF method. The structure-activity relationship was discussed and the possible mechanism was suggested. It could provide guidance for configuration of these compounds to improve the biological activity.
\end{abstract}

Key Words: Fluoride pyrazole, Diacylhydrazine, Biological activity, Structure-activity relationship.

\section{INTRODUCTION}

In search of novel bioactive compounds, 19 compounds were designed and synthesized by introducing the fluoropyrazolyl pharmacophore into the diacylhydrazine scaffold by Zhang et al. ${ }^{1}$ and others ${ }^{2-5}$. The herbicidal activities of these compounds were evaluated. The bioassay results showed that most of them exhibited higher herbicidal activities against dicotyledonous weeds (Abutilon theophrasti, Amaranthus ascedense and Amaranthus retroflexus) than monocotyledonous weeds.

The quantum study of diacylhydrazine derivatives fluoridecontaining pyrazolyl moiety was calculated by Gaussian 03 program. The study found the correlation between the herbicidal activity of these compounds and structural parameters and filter the main factors to affect the biological activity and the influence to biological activity from the changes in the molecular structure was explained, the mechanism and sites of action of compound was also discussed ${ }^{6,7}$.

\section{EXPERIMENTAL}

The geometries of all compounds were optimized using the ab initio HF with the 6-31G* basis set. Harmonic vibrational frequencies calculated at the same level were used for characterization of stationary points as a minimum. All quantum calculations were performed with the Gaussian 03 program.

\section{RESULTS AND DISCUSSION}

Stability configurations and natural charge: The structure of compounds as follows:

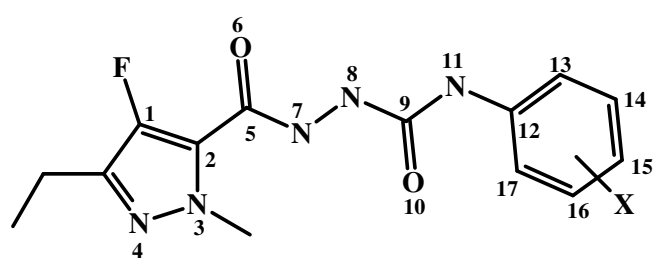

I: $\mathrm{X}=$ (3a)16-C; (3c)16-H; (3g)13-C; (3h)15-C; (3k)15-C, 17-C; (3l) 14C, 15-C; (3n)13-C, 17-C; (3o)14-C, 17-C; (3p)14-C, 16-C, II: X= (3b)15$\mathrm{Cl}$; (3i) 13-Cl; (3j)14-Cl, III: X = (3m)13-F, 15-F; (3q)16-F, 17-F; (3r)13F, 16-F; (3s)13-F, 17-F, IV: X = (3d)13- $\mathrm{NO}_{2}$; (3e)15- $\mathrm{NO}_{2} ;$ (3f) $14-\mathrm{NO}_{2}$

Fig. 1. Structure of compounds

Energy of the frontier molecules orbital: According to the theory of molecular orbital (MO), the highest occupied molecular orbital (HOMO) and the lowest unoccupied molecular orbital (LUMO) have the greatest influence on the activity of compounds. The reaction between active molecule and receptor macromolecular operated on the frontier molecules orbital. $\mathrm{E}_{\text {Hомо }}$ is the energy of HOMO, which relate to the ability of electron donor. $\mathrm{E}_{\mathrm{LUMO}}$ is the energy of LUMO, which relate to the ability of acceptance of electronic. For pesticide molecules, too low- $\mathrm{E}_{\mathrm{Lumo}}$ or too high- $\mathrm{E}_{\text {номо means }}$ that the molecule itself activity is too strong, it is easy to be metabolized in organism, The effect of pesticide is difficult to control, so the $\mathrm{E}_{\mathrm{LUmO}}$ or $\mathrm{E}_{\text {номо }}$ of the pesticide molecule should be suitable to estimate expected value ${ }^{8-10}$.

From Table-1, the data of herbicidal activity is the best of compound $\mathbf{3 h}, \mathbf{3 k}$ and $\mathbf{3 l}$, the $\mathrm{E}_{\text {номо }}$ of $\mathbf{3 h}, \mathbf{3 k}$ and $\mathbf{3 l}$ is the higher, so it has strong ability to provide electron and the data 
TABLE-1

ENERGY OF THE FRONTIER MOLECULES ORBITALS

\begin{tabular}{|c|c|c|c|c|c|c|c|}
\hline Compound & $\mathrm{E}_{\text {номо }}$ & $\mathrm{E}_{\text {LUMO }}$ & $\Delta \mathrm{E}$ & Compound & $\mathrm{E}_{\text {НОмо }}$ & $\mathrm{E}_{\text {LUMO }}$ & $\Delta \mathrm{E}$ \\
\hline $\mathbf{3 a}$ & -8.2051 & 2.7680 & 10.9731 & $3 \mathbf{i}$ & -8.6122 & 2.7688 & 11.3810 \\
\hline 3c & -8.3134 & 2.7530 & 11.0664 & $\mathbf{3 j}$ & -8.6326 & 2.6433 & 11.2759 \\
\hline $3 g$ & -8.2914 & 2.7574 & 11.0487 & $3 m$ & -9.2574 & 2.7261 & 11.9834 \\
\hline $3 h$ & -8.0789 & 2.7756 & 10.8544 & $3 q$ & -9.3213 & 2.7070 & 12.0283 \\
\hline $3 \mathbf{k}$ & -8.0797 & 2.7810 & 10.8607 & $3 r$ & -9.0789 & 2.7103 & 11.7892 \\
\hline 31 & -7.9904 & 2.7876 & 10.7780 & $3 s$ & -9.3113 & 2.8218 & 12.1331 \\
\hline $3 n$ & -8.6900 & 2.8191 & 11.5091 & 3d & -9.0715 & 1.5655 & 10.6370 \\
\hline 30 & -8.1814 & 2.7704 & 10.9519 & $3 \mathbf{e}$ & -8.6670 & 2.4140 & 11.0810 \\
\hline $3 p$ & -8.1532 & 2.7813 & 10.9336 & 3f & -8.9380 & 1.6710 & 10.609 \\
\hline $3 \mathbf{b}$ & -8.4478 & 2.6373 & 11.0852 & - & - & - & - \\
\hline
\end{tabular}

of herbicidal activity is not good for compound $\mathbf{3 m}, \mathbf{3 q}$ and $\mathbf{3 s}$, the $\mathrm{E}_{\text {номо }}$ of $\mathbf{3 m}, \mathbf{3 q}$ and $\mathbf{3 s}$ is low, so it has low ability to provide electron. It is concluded that the higher $\mathrm{E}_{\text {Номо }}$ of compound, relatively better herbicidal activity of the compound. And the conclusion is consistent with the experimental values. This may be the reason of these compounds to served as herbicidals. Therefore, the mechanism is that herbicidal molecule provide electronic to receptor possibly.

Main composition and proportion of the frontier molecules orbital: From Table-2, the main composition and proportion of $\mathrm{E}_{\text {Номо }}$ of compounds I, II and IV in the N(8), $\mathrm{O}(10), \mathrm{N}(11)$ and the $\mathrm{C}$ of benzene ring. The main composition and proportion of $\mathrm{E}_{\mathrm{Hомо}}$ of them in the pyrazole ring and diacylhydrazine. The main composition and proportion of

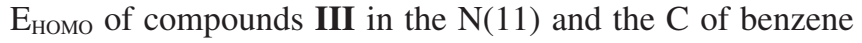
ring and the composition is low of $\mathrm{N}(8)$ and $\mathrm{O}(10)$, this is one of the reasons for the difference of the herbicidal activity.

Natural charge: The atom natural charge of compounds is given in Table- 3 . These data show that, the negative charge is mainly concentrated in the $\mathrm{N}(8), \mathrm{O}(10)$ and $\mathrm{N}(11)$ of

\begin{tabular}{|c|c|c|c|}
\hline \multicolumn{4}{|c|}{$\begin{array}{l}\text { TABLE-2 } \\
\text { MAIN COMPOSITION AND PROPORTION OF FRONTIER MOLECULES ORBITAL }\end{array}$} \\
\hline Com & ound & HOMO & $\begin{array}{r}\text { LUMO } \\
\end{array}$ \\
\hline \multirow{9}{*}{$\mathbf{I}$} & $\mathbf{3 a}$ & $\begin{array}{l}\mathrm{N}(8) 1.42 ; \mathrm{O}(10) 2.62 ; \mathrm{N}(11) 19.62 ; \mathrm{C}(12) 20.02 ; \mathrm{C}(13) 7.02 \\
\mathrm{C}(14) 2.07 ; \mathrm{C}(15) 25.19 ; \mathrm{C}(16) 14.07 ; \mathrm{C}(17) 4.94\end{array}$ & $\begin{array}{c}\mathrm{C}(1) 11.69 ; \mathrm{C}(2) 11.06 ; \mathrm{N}(3) 12.28 ; \mathrm{N}(4) 11.34 ; \mathrm{C}(5) 19.41 ; \\
\mathrm{O}(6) 15.18 ; \mathrm{N}(7) 5.63 ; \mathrm{N}(8) 2.63\end{array}$ \\
\hline & $3 \mathbf{c}$ & $\begin{array}{l}\mathrm{N}(8) 1.50 ; \mathrm{O}(10) 2.98 ; \mathrm{N}(11) 21.39 ; \mathrm{C}(12) 20.94 ; \mathrm{C}(13) 9.83 \\
\mathrm{C}(14) 3.69 ; \mathrm{C}(15) 24.09 ; \mathrm{C}(16) 4.61 ; \mathrm{C}(17) 9.25\end{array}$ & $\begin{array}{c}\mathrm{C}(1) 11.70 ; \mathrm{C}(2) 11.04 ; \mathrm{N}(3) 15.18 ; \mathrm{N}(4) 11.33 ; \mathrm{C}(5) 19.44 ; \\
\mathrm{O}(6) 12.31 ; \mathrm{N}(7) 5.62 ; \mathrm{N}(8) 2.63\end{array}$ \\
\hline & $3 g$ & $\begin{array}{l}\mathrm{N}(8) 1.20 ; \mathrm{O}(10) 2.75 ; \mathrm{N}(11) 19.40 ; \mathrm{C}(12) 22.98 ; \mathrm{C}(13) 12.10 \\
\mathrm{C}(14) 2.42 ; \mathrm{C}(15) 22.73 ; \mathrm{C}(16) 6.23 ; \mathrm{C}(17) 6.37\end{array}$ & $\begin{array}{c}\mathrm{C}(1) 11.64 ; \mathrm{C}(2) 11.17 ; \mathrm{N}(3) 15.25 ; \mathrm{N}(4) 11.27 ; \mathrm{C}(5) 19.19 ; \\
\mathrm{O}(6) 12.17 ; \mathrm{N}(7) 5.61 ; \mathrm{N}(8) 2.43\end{array}$ \\
\hline & $3 \mathbf{h}$ & $\begin{array}{l}\mathrm{N}(8) 1.31 ; \mathrm{O}(10) 2.65 ; \mathrm{N}(11) 18.90 ; \mathrm{C}(12) 20.75 ; \mathrm{C}(13) 8.25 \\
\mathrm{C}(14) 4.85 ; \mathrm{C}(15) 23.14 ; \mathrm{C}(16) 5.62 ; \mathrm{C}(17) 8.39\end{array}$ & $\begin{array}{c}\mathrm{C}(1) 11.68 ; \mathrm{C}(2) 11.08 ; \mathrm{N}(3) 15.19 ; \mathrm{N}(4) 11.34 ; \mathrm{C}(5) 19.39 \\
\mathrm{O}(6) 12.28 ; \mathrm{N}(7) 5.63 ; \mathrm{N}(8) 2.60\end{array}$ \\
\hline & $3 \mathbf{k}$ & $\begin{array}{l}\mathrm{N}(8) 1.05 ; \mathrm{O}(10) 2.43 ; \mathrm{N}(11) 17.08 ; \mathrm{C}(12) 23.03 ; \mathrm{C}(13) 5.96 \\
\mathrm{C}(14) 6.94 ; \mathrm{C}(15) 22.03 ; \mathrm{C}(16) 3.86 ; \mathrm{C}(17) 9.95\end{array}$ & $\begin{array}{c}\mathrm{C}(1) 11.68 ; \mathrm{C}(2) 11.26 ; \mathrm{N}(3) 15.34 ; \mathrm{N}(4) 11.34 ; \mathrm{C}(5) 19.20 ; \\
\mathrm{O}(6) 5.64 ; \mathrm{N}(7) 12.16 ; \mathrm{N}(8) 2.39\end{array}$ \\
\hline & 31 & $\begin{array}{l}\mathrm{N}(8) 1.28 ; \mathrm{O}(10) 2.40 ; \mathrm{N}(11) 17.73 ; \mathrm{C}(12) 19.92 ; \mathrm{C}(13) 4.84 ; \\
\mathrm{C}(14) 7.33 ; \mathrm{C}(15) 24.05 ; \mathrm{C}(16) 3.43 ; \mathrm{C}(17) 11.71\end{array}$ & $\begin{array}{c}\mathrm{C}(1) 11.68 ; \mathrm{C}(2) 11.09 ; \mathrm{N}(3) 15.20 ; \mathrm{N}(4) 11.35 ; \mathrm{C}(5) 19.36 \\
\mathrm{O}(6) 12.25 ; \mathrm{N}(7) 5.64 ; \mathrm{N}(8) 2.61\end{array}$ \\
\hline & $3 n$ & $\mathrm{C}(13) 21.87 ; \mathrm{C}(14) 26.10 ; \mathrm{C}(16) 23.97 ; \mathrm{C}(17) 19.42$ & $\begin{array}{c}\mathrm{C}(1) 11.55 ; \mathrm{C}(2) 11.41 ; \mathrm{N}(3) 15.39 ; \mathrm{N}(4) 11.33 ; \mathrm{C}(5) 18.88 \\
\mathrm{O}(6) 11.94 ; \mathrm{N}(7) 5.60 ; \mathrm{N}(8) 2.57\end{array}$ \\
\hline & 30 & $\begin{array}{c}\mathrm{N}(8) 0.99 ; \mathrm{O}(10) 2.39 ; \mathrm{N}(11) 16.35 ; \mathrm{C}(12) 19.84 ; \mathrm{C}(13) 17.30 \\
\mathrm{C}(14) 10.43 ; \mathrm{C}(15) 23.15 ; \mathrm{C}(17) 2.18\end{array}$ & $\begin{array}{c}\mathrm{C}(1) 11.68 ; \mathrm{C}(2) 11.22 ; \mathrm{N}(3) 15.31 ; \mathrm{N}(4) 11.32 ; \mathrm{C}(5) 19.25 \\
\mathrm{O}(6) 12.20 ; \mathrm{N}(7) 5.63 ; \mathrm{N}(8) 2.45\end{array}$ \\
\hline & $3 p$ & $\begin{array}{l}\mathrm{N}(8) 1.38 ; \mathrm{O}(10) 2.66 ; \mathrm{N}(11) 19.29 ; \mathrm{C}(12) 19.56 ; \mathrm{C}(13) 11.29 \\
\mathrm{C}(14) 3.40 ; \mathrm{C}(15) 26.99 ; \mathrm{C}(16) 5.04 ; \mathrm{C}(17) 6.82\end{array}$ & $\begin{array}{c}\mathrm{C}(1) 11.69 ; \mathrm{C}(2) 11.07 ; \mathrm{N}(3) 15.19 ; \mathrm{N}(4) 11.35 ; \mathrm{C}(5) 19.38 \\
\mathrm{O}(6) 12.26 ; \mathrm{N}(7) 5.64 ; \mathrm{N}(8) 2.63\end{array}$ \\
\hline \multirow{3}{*}{ II } & $3 \mathbf{b}$ & $\begin{array}{l}\mathrm{N}(8) 1.24 ; \mathrm{O}(10) 2.34 ; \mathrm{N}(11) 17.54 ; \mathrm{C}(12) 17.58 ; \mathrm{C}(13) 7.52 \\
\mathrm{C}(14) 4.47 ; \mathrm{C}(15) 21.09 ; \mathrm{C}(16) 3.86 ; \mathrm{C}(17) 7.61 ; \mathrm{Cl}(\mathrm{X}) 15.32\end{array}$ & $\begin{array}{c}\mathrm{C}(1) 11.71 ; \mathrm{C}(2) 10.85 ; \mathrm{N}(3) 15.05 ; \mathrm{N}(4) 11.20 ; \mathrm{C}(5) 19.61 ; \\
\mathrm{O}(6) 12.42 ; \mathrm{N}(7) 5.57 ; \mathrm{N}(8) 2.71\end{array}$ \\
\hline & $3 \mathbf{i}$ & $\begin{array}{l}\mathrm{N}(8) 1.53 ; \mathrm{O}(10) 2.83 ; \mathrm{N}(11) 21.05 ; \mathrm{C}(12) 19.26 ; \mathrm{C}(13) 12.82 \\
\mathrm{C}(14) 2.48 ; \mathrm{C}(15) 20.87 ; \mathrm{C}(16) 4.97 ; \mathrm{C}(17) 6.25 ; \mathrm{Cl}(\mathrm{X}) 6.25\end{array}$ & $\begin{array}{c}\mathrm{C}(1) 11.62 ; \mathrm{C}(2) 10.97 ; \mathrm{N}(3) 15.15 ; \mathrm{N}(4) 11.37 ; \mathrm{C}(5) 19.32 ; \\
\mathrm{O}(6) 12.21 ; \mathrm{N}(7) 5.60 ; \mathrm{N}(8) 2.76\end{array}$ \\
\hline & $\mathbf{3 j}$ & $\begin{array}{c}\mathrm{N}(8) 1.36 ; \mathrm{O}(10) 2.81 ; \mathrm{N}(11) 19.88 ; \mathrm{C}(12) 17.44 ; \mathrm{C}(13) 4.79 \\
\mathrm{C}(14) 6.78 ; \mathrm{C}(15) 24.00 ; \mathrm{C}(17) 14.00 ; \mathrm{Cl}(\mathrm{X}) 5.72\end{array}$ & $\begin{array}{c}\mathrm{C}(1) 11.72 ; \mathrm{C}(2) 10.84 ; \mathrm{N}(3) 15.04 ; \mathrm{N}(4) 11.20 ; \mathrm{C}(5) 19.62 ; \\
\mathrm{O}(6) 12.42 ; \mathrm{N}(7) 5.57 ; \mathrm{N}(8) 2.71\end{array}$ \\
\hline \multirow{4}{*}{ III } & $3 \mathbf{m}$ & $\begin{array}{c}\mathrm{N}(11) 7.76 ; \mathrm{C}(12) 27.16 ; \mathrm{C}(13) 12.99 ; \mathrm{C}(15) 16.87 ; \mathrm{C}(16) 18.69 ; \\
\mathrm{F}\left(\mathrm{X}_{1}\right) 4.52 ; \mathrm{F}\left(\mathrm{X}_{2}\right) 6.23\end{array}$ & $\begin{array}{c}\mathrm{C}(1) 11.68 ; \mathrm{C}(2) 11.43 ; \mathrm{N}(3) 15.38 ; \mathrm{N}(4) 11.22 ; \mathrm{C}(5) 19.05 ; \\
\mathrm{O}(6) 12.16 ; \mathrm{N}(7) 5.61 ; \mathrm{N}(8) 2.45\end{array}$ \\
\hline & $3 q$ & $\begin{array}{c}\mathrm{N}(11) 3.17 ; \mathrm{C}(12) 6.99 ; \mathrm{C}(13) 8.62 ; \mathrm{C}(14) 24.16 ; \mathrm{C}(15) 3.25 \\
\mathrm{C}(16) 11.13 ; \mathrm{C}(17) 27.45 ; \mathrm{F}\left(\mathrm{X}_{1}\right) 9.52 ; \mathrm{F}\left(\mathrm{X}_{2}\right) 3.69\end{array}$ & $\begin{array}{c}\mathrm{C}(1) 11.63 ;(2) 11.39 ; \mathrm{N}(3) 15.33 ; \mathrm{N}(4) 11.15 ; \mathrm{C}(5) 19.03 ; \\
\mathrm{O}(6) 5.60 ; \mathrm{N}(7) 12.17 ; \mathrm{N}(8) 2.40\end{array}$ \\
\hline & $3 \mathbf{r}$ & $\begin{array}{c}\mathrm{N}(11) 5.81 ; \mathrm{C}(12) 14.07 ; \mathrm{C}(13) 22.83 ; \mathrm{C}(14) 3.73 ; \mathrm{C}(15) 13.25 \\
\mathrm{C}(16) 20.11 ; \mathrm{C}(17) 2.60 ; \mathrm{F}\left(\mathrm{X}_{1}\right) 7.81 ; \mathrm{F}\left(\mathrm{X}_{2}\right) 7.09\end{array}$ & $\begin{array}{c}\mathrm{C}(1) 11.55 ; \mathrm{C}(2) 11.20 ; \mathrm{N}(3) 15.13 ; \mathrm{N}(4) 11.02 ; \mathrm{C}(5) 19.01 ; \\
\mathrm{O}(6) 12.12 ; \mathrm{N}(7) 5.54 ; \mathrm{N}(8) 2.39\end{array}$ \\
\hline & $3 \mathbf{s}$ & $\begin{array}{c}\mathrm{N}(11) 14.43 ; \mathrm{C}(12) 26.15 ; \mathrm{C}(13) 13.81 ; \mathrm{C}(15) 17.18 ; \mathrm{C}(16) 14.04 ; \\
\mathrm{F}\left(\mathrm{X}_{1}\right) 4.83\end{array}$ & $\begin{array}{c}\mathrm{C}(1) 11.47 ; \mathrm{C}(2) 11.08 ; \mathrm{N}(3) 15.16 ; \mathrm{N}(4) 11.28 ; \mathrm{C}(5) 19.12 \\
\mathrm{O}(6) 12.11 ; \mathrm{N}(7) 5.60 ; \mathrm{N}(8) 2.39\end{array}$ \\
\hline \multirow{3}{*}{ IV } & 3d & $\begin{array}{l}\mathrm{N}(8) 1.83 ; \mathrm{O}(10) 2.61 ; \mathrm{N}(11) 23.35 ; \mathrm{C}(12) 15.78 ; \mathrm{C}(13) 6.52 ; \\
\mathrm{C}(14) 9.44 ; \mathrm{C}(15) 22.34 ; \mathrm{C}(16) 1.52 ; \mathrm{C}(17) 14.12\end{array}$ & $\begin{array}{c}\mathrm{C}(12) 11.12 ; \mathrm{C}(13) 10.11 ; \mathrm{C}(14) 10.27 ; \mathrm{C}(16) 15.32 ; \mathrm{C}(17) 2.07 ; \\
\mathrm{N}(\mathrm{X}) 20.24 ; \mathrm{O}(\mathrm{X} 1) 12.23 ; \mathrm{O}(\mathrm{X} 2) 13.19\end{array}$ \\
\hline & $3 \mathbf{e}$ & $\begin{array}{c}\mathrm{N}(8) 1.71 ; \mathrm{O}(10) 2.13 ; \mathrm{N}(11) 23.33 ; \mathrm{C}(12) 16.56 ; \mathrm{C}(13) 11.09 \\
\mathrm{C}(14) 1.84 ; \mathrm{C}(15) 24.74 ; \mathrm{C}(16) 2.15 ; \mathrm{C}(17) 9.76 ; \mathrm{O}\left(\mathrm{X}_{1}\right) 2.20 \\
\mathrm{O}\left(\mathrm{X}_{2}\right) 2.89\end{array}$ & $\begin{array}{c}\mathrm{C}(9) 2.62 ; \mathrm{N}(11) 1.84 ; \mathrm{C}(12) 20.43 ; \mathrm{C}(14) 11.86 ; \mathrm{C}(15) 13.78 ; \\
\mathrm{C}(16) 11.01 ; \mathrm{N}(\mathrm{X}) 19.70 ; \mathrm{O}(\mathrm{X} 1) 13.31\end{array}$ \\
\hline & $3 \mathbf{f}$ & $\begin{array}{c}\mathrm{O}(10) 3.18 ; \mathrm{N}(11) 24.34 ; \mathrm{C}(12) 19.60 ; \mathrm{C}(13) 12.70 ; \mathrm{C}(14) 3.50 \\
\mathrm{C}(15) 20.64 ; \mathrm{C}(16) 3.96 ; \mathrm{C}(17) 7.33\end{array}$ & $\begin{array}{c}\mathrm{C}(13) 11.93 ; \mathrm{C}(14) 14.41 ; \mathrm{C}(15) 9.08 ; \mathrm{C}(16) 2.41 ; \mathrm{C}(17) 16.08 ; \\
\mathrm{N}(\mathrm{X}) 18.53 ; \mathrm{O}(\mathrm{X} 1) 12.91 ; \mathrm{O}(\mathrm{X} 2) 12.53\end{array}$ \\
\hline
\end{tabular}




\begin{tabular}{|c|c|c|c|c|c|c|c|c|c|}
\hline \multicolumn{10}{|c|}{$\begin{array}{c}\text { TABLE-3 } \\
\text { ATOM NATURAL CHARGE OF CON }\end{array}$} \\
\hline \multicolumn{2}{|c|}{ Compound } & $\mathrm{C}(1)$ & $\mathrm{C}(5)$ & $\mathrm{O}(6)$ & $\mathrm{N}(7)$ & $\mathrm{N}(8)$ & $\mathrm{C}(9)$ & $\mathrm{O}(10)$ & $\mathrm{N}(11)$ \\
\hline \multirow{9}{*}{ I } & $3 a$ & 0.41496 & 0.82737 & -0.68694 & -0.55891 & -0.55823 & 1.01201 & -0.74829 & -0.72018 \\
\hline & $3 c$ & 0.41489 & 0.82732 & -0.68644 & -0.55903 & -0.5581 & 1.01196 & -0.74868 & -0.7209 \\
\hline & $3 g$ & 0.41349 & 0.8274 & -0.68767 & -0.55795 & -0.55874 & 1.01207 & -0.7475 & -0.73183 \\
\hline & $3 \mathbf{h}$ & 0.4147 & 0.82732 & -0.68696 & -0.55871 & -0.55822 & 1.01144 & -0.75001 & -0.72059 \\
\hline & $3 k$ & 0.4136 & 0.82748 & -0.68862 & -0.55754 & -0.55885 & 1.0116 & -0.74875 & -0.73228 \\
\hline & 31 & 0.41484 & 0.82738 & -0.68743 & -0.55866 & -0.55833 & 1.01154 & -0.74951 & -0.71962 \\
\hline & $3 n$ & 0.41344 & 0.8268 & -0.69055 & -0.55418 & -0.55848 & 1.00856 & -0.74859 & -0.75503 \\
\hline & 30 & 0.41352 & 0.82743 & -0.68777 & -0.55785 & -0.55885 & 1.01231 & -0.74776 & -0.73092 \\
\hline & $3 p$ & 0.41506 & 0.82741 & -0.68726 & -0.55876 & -0.55834 & 1.01221 & -0.74851 & -0.71976 \\
\hline \multirow{3}{*}{ II } & $3 b$ & 0.41465 & 0.82705 & -0.68384 & -0.56022 & -0.55728 & 1.01272 & -0.74752 & -0.72085 \\
\hline & $3 \mathbf{i}$ & 0.41617 & 0.82831 & -0.6852 & -0.55807 & -0.55587 & 1.01521 & -0.74866 & -0.72786 \\
\hline & $3 \mathbf{j}$ & 0.41472 & 0.82708 & -0.68386 & -0.56029 & -0.55726 & 1.01272 & -0.74446 & -0.72084 \\
\hline \multirow{4}{*}{ III } & $3 m$ & 0.40994 & 0.8265 & -0.68603 & -0.55616 & -0.55897 & 1.01313 & -0.73943 & -0.75006 \\
\hline & $3 q$ & 0.40886 & 0.82602 & -0.68469 & -0.55571 & -0.55868 & 1.01277 & -0.7336 & -0.74951 \\
\hline & $3 r$ & 0.40923 & 0.82608 & -0.68458 & -0.55581 & -0.55862 & 1.01309 & -0.73364 & -0.74878 \\
\hline & $3 s$ & 0.41431 & 0.82657 & -0.68778 & -0.55419 & -0.55723 & 1.01448 & -0.73518 & -0.74441 \\
\hline \multirow{3}{*}{ IV } & 3d & 0.41554 & 0.82963 & -0.6827 & -0.55419 & -0.55365 & 1.01562 & -0.74554 & -0.73869 \\
\hline & $3 e$ & 0.41474 & 0.82665 & -0.67979 & -0.56203 & -0.55622 & 1.0146 & -0.73991 & -0.72088 \\
\hline & $3 f$ & 0.41479 & 0.8267 & -0.67982 & -0.56117 & -0.5561 & 1.01361 & -0.74767 & -0.72199 \\
\hline
\end{tabular}

acylhydrazine, these atoms make the electronegative area and they could combine with positive area of receptor. the positive charge is mainly concentrated in the $\mathrm{C}(1)$ of carbon-fluorine bond and $\mathrm{C}(5), \mathrm{C}(9)$ of carbonyl, These atoms make the positive area, they could combine with negative area of receptor.

The groups (-Cl, $\left.-\mathrm{F},-\mathrm{NO}_{2}\right)$ of II, III and IV are electronwithdrawing groups, they conjugate with benzene ring and reduces the density of electron of benzene ring. The group $\left(-\mathrm{CH}_{3}\right)$ of $\mathbf{I}$ is electron-donating group, it conjugate with benzene ring, it increases the density of electron of benzene ring. The I provide electronic to receptor easier than II, III and $\mathbf{I V}$, this is one of the reasons for the difference of the herbicidal activity. For example, the herbicidal activity of $\mathbf{3 k}$ and $\mathbf{3 l}$ is better than $\mathbf{3 d}, \mathbf{3} \mathbf{j}$ and $\mathbf{3 s}$.

\section{Conclusion}

The characteristics of HOMO and LUMO are the main factors to influence antibacterial activities of these kinds of compounds. The mechanism is that diacylhydrazine derivatives fluoride-containing pyrazolyl moiety provide electronic to the receptor. The results indicate that acylhydrazine might be an important active site, of which the potency of electric charge translocation has a great influence on the herbicidal activity of this kind of compounds.

\section{ACKNOWLEDGEMENTS}

This project was supported by the Science and Technology Projects of Hebei Province (Contract No. 10273939) and Handan Key Laboratory of Organic Small Molecule Materials, Handan College.

\section{REFERENCES}

1. X.Y. Zhang, Y.S. Li, J.Q. Weng and C.X. Tan, Chin. J. Org. Chem., 31, 1295 (2011)

2. S.L. Labhade and V.B. Gaikwad, Asian J. Chem., 21, 7117 (2009).

3. J.J. Heller, H. Mattioda, E. Klein and A. Sagenmuller, Brighton Crop Protection Conference Pests and Diseases, Brighton, 1, 59 (2000).

4. X.H. Liu, Y.X. Shi, Y. Ma, C.Y. Zhang, W.L. Dong, P. Li, B.L. Wang, B.J. Li and Z.M. Li, Eur. J. Med. Chem., 44, 2782 (2009).

5. X.H. Liu, Y.X. Shi, Y. Ma, G.R. He, W.L. Dong, C.Y. Zhang, B.L. Wang, S.H. Wang, B.J. Li and Z.M. Li, Chem. Biol. Drug Des., 73, 320 (2009).

6. G.C.L. Ee, S.H. Teo, R. Go, C.K. Lim, Y.M. Lim and C.F.J. Bong, Asian J. Chem., 24, 231 (2012).

7. B.K. Sharma, Yashwant and B. Srivastava, Asian J. Chem., 22, 8231 (2010).

8. T.B. Wei, Y.L. Leng, Y.C. Wang, J.H. Zhang and Y.M. You, Chin. J. Org. Chem., 29, 216 (2009).

9. G.F. Yang, H.Y. Liu, Z.H. Yang and X.F. Yang, Acta Chim. Sin., 56, 729 (1998).

10. D.B. Zhang, Y.H. Ren, D.W. Fu, B. Yan, J.R. Song and X.Q. Lv, Acta Chim. Sin., 66, 2409 (2008). 